\title{
Fusion of Sun-Synchronous and Geostationary Images for Coastal and Ocean Color Survey Application to OLCI (Sentinel-3) and FCl (MTG)
}

\author{
Peschoud Cecile ${ }^{1,2}$, Minghelli Audrey ${ }^{1,2}$, Mathieu Sandrine ${ }^{3}$, Lei Manchun ${ }^{1,2}$, Pairaud Ivane ${ }^{4}$, \\ Pinazo Christel ${ }^{5,6}$
}

${ }^{1}$ Univ Toulon \& Var, LSIS, CNRS, UMR 7296, Toulon 9, France.

${ }^{2}$ Aix Marseille Univ, CNRS, ENSAM, LSIS UMR 7296 13397, Marseille, France.

${ }^{3}$ Thales Alenia Space, F-06156 Cannes La Bocca, France.

${ }^{4}$ IFREMER, LERPAC, Ctr Mediterranee, Zone Portuaire Bregaillon CS 20 330, F-83507 La Seyne Sur Mer, France.

${ }^{5}$ Aix Marseille Univ, CNRS, INSU, IRD,MIO,UM110, F-13288 Marseille, France.

${ }^{6}$ Univ deToulon, CNRS, INSU, IRD,MIO,UM110, F-83957 La Garde, France.

Corresponding author email address : cecile.peschoud@univ-tln.fr ; audrey.minghelli@univ-tln.fr ; sandrine.mathieu@thalesaleniaspace.com ; manchun.lei@univ-tIn.fr ; ivane.pairaud@ifremer.fr

\begin{abstract}
:
Open ocean and coastal area monitoring requires multispectral satellite images with a middle spatial resolution (\{\sim !300 \{text\{m\}\}\}) and a high temporal repeatability (\{\sim !1 \{text\{h\}\}\}). As no current satellite sensors have such features, the aim of this study is to propose a fusion method to merge images delivered by a low earth orbit (LEO) sensor with images delivered by a geostationary earth orbit (GEO) sensor. This fusion method, called spatial spectral temporal fusion (SSTF), is applied to the future sensors-Ocean and Land Color Instrument (OLCI) (on Sentinel-3) and Flexible Combined Imager (FCI) (on Meteosat Third Generation) whose images were simulated. The $\mathrm{OLCl}$ bands, acquired at $\mathrm{t}\{0\}$, are divided by the oversampled corresponding $\mathrm{FCl}$ band acquired at $\mathrm{t} \_\{0\}$ and multiplied by the $\mathrm{FCl}$ bands acquired at $\mathrm{t} \_\{1\}$. The fusion product is used for the next fusion at $\mathrm{t} \_\{1\}$ and so on. The high temporal resolution of $\mathrm{FCl}$ allows its signal-tonoise ratio (SNR) to be enhanced by the means of temporal filtering. The fusion quality indicator ERGAS computed between SSTF fusion products and reference images is around 0.75 , once the $\mathrm{FCl}$ images are filtered from the noise and 1.08 before filtering. We also compared the estimation of chlorophyll (Chl), suspended particulate matter (SPM), and colored dissolved organic matter (CDOM) maps from the fusion products with the input simulation maps. The comparison shows an average relative errors on Chl, SPM, and CDOM, respectively, of $64.6 \%, 6.2 \%$, and $9.5 \%$ with the SSTF method. The SSTF method was also compared with an existing fusion method called the spatial and temporal adaptive reflectance fusion model (STARFM).
\end{abstract}

Keywords : Fusion, image simulation, meteosat Third Generation (MTG), ocean color, Ocean and Land Color Instrument 


\section{Introduction}

Many problems of natural or anthropogenic origin are present in offshore and coastal waters. Remote sensing allows some of these phenomena to be detected or monitored directly or indirectly. Water color has been used for a long time to determine the water composition in terms of Chl, SPM and $C D O M$ in open water and more recently in coastal areas. New applications have emerged thanks to the increase of the number of spectral bands, the radiometric measurement quality and the observation frequency [1].

For the ocean survey, many sensors have been developed over the past thirty years such as CZCS [2], SeaWiFS [3], MODIS [4], MERIS [5], VIIRS [6] and the future Ocean and Land Color Instrument (OLCI) [7]. The spatial, spectral and radiometric resolutions of these sensors increased over the time $(300 \mathrm{~m}, 21$ bands and SNR 1200 for OLCI) but, due to their positioning in LEO orbit, their temporal repetitivity remains low with at best one daily revisit (when using 3 to 4 satellites).

As one daily revisit is not sufficient, for high temporal dynamics ecosystems like coastal areas, the geostationary sensors are becoming increasingly considered for the water color survey [8], [9]. Their spatial and spectral resolutions are often limited for the coastal area needs, but they can provide almost continuous images over a same area. Meteosat is the only existing geostationary satellite covering Europe and Africa. Some studies on coastal waters survey with geostationary sensors proved the feasibility of SPM mapping as with the Spinning Enhanced Visible and InfraRed Imager (SEVIRI) on Meteosat Second Generation (MSG) but its spatial and spectral resolutions were still limited [10], [11]. The Geostationary Ocean Color Imager (GOCI) onboard COMS satellite provides images with higher spatial and spectral resolutions $(500 \mathrm{~m}, 8$ spectral bands in the visible range) every hour but it covers only a limited area around the Korean Peninsula [12]. We are thus interested in merging the images provided by a sun-synchronous and a geostationary sensor in order to get the required information to detect and monitor marine phenomena.

Many fusion methods have already been developed. Some of these methods such as ARSIS (from its French acronym "Amélioration de la Résolution Spatiale par Injection de Structure") [13] enables the spatial resolution to be improved by the structure injection, using a multiresolution analysis approach. Methods were also developed to merge several multispectral images such as MMT (Multisensor Multiresolution Technique) inspired from Zhukov et al. [14]. MinghelliRoman et al. [15], [16] implemented this method to sharpen a MERIS image with the spatial information from an ETM (Landsat) image. Most recently, Yokoya et al. [17] developed a method called CNMF (Coupled Nonnegative Matrix Factorization) to fuse multispectral and hyperspectral images by decomposing the hyperspectral input image into an endmember's spectra matrix and the multispectral image into an abundance matrix. Sylla et al. [18] also merged multispectral images (MSI on Sentinel-2 and OLCI on Sentinel-3) using a method adapted from ARSIS [17]. But all these methods assume that the landscape does not change between the 2 acquisitions. The temporal variations of the landscape between the two image acquisitions is however taken into account by very few fusion methods, such as STARFM (Spatial and Temporal Adaptive Reflectance Fusion Model) [19]. It was implemented to fuse MODIS and Landsat images over forest areas and showed accurate detection of phenology changes. Q. Vanhellemont [20] also developed a method to combine data from the single broad red band of the SEVIRI geostationary sensor with the 
corresponding band of MODIS sun-synchronous sensor. This process enables the high spatial information provided by the sun-synchronous sensor (MODIS) to be merged with the high temporal information provided by the geostationary sensor (SEVIRI) but it is applied only to one band.

The originality of this paper is the extension of the method developed by Q. Vanhellemont [20] to fuse images provided by a multispectral LEO sensor and by a multispectral GEO sensor to obtain images having the spatial resolution of the LEO sensor and the spectral and temporal resolution of the GEO sensor to finally obtain a reliable water quality indicator. We called this method the Spatial Spectral Temporal Fusion method (SSTF).

The SSTF method is applied to 2 future sensors: the next European ocean color sensor, OLCI on Sentinel-3, planned to fulfill a mission in the continuity of ENVISAT/MERIS sensor with 6 more spectral bands and an improved SNR. The second sensor belongs to the next generation of European meteorological satellites, Meteosat Third Generation (MTG) with the onboard Flexible Combined Imager (FCI) which is dedicated mainly to meteorological survey. Planned to follow on from MSG/SEVIRI, MTG/FCI will provide images every 10 minutes with a spatial resolution of 1 $\mathrm{km}$ on 5 VIS spectral bands except for the $3^{\text {rd }}$ band that reaches a $0.5 \mathrm{~km}$ spatial resolution.

At the time this paper was being written, the two sensors were under development at Thales Alenia Space, we thus describe in this paper the process to provide OLCI and FCI simulated images. In order to artificially increase FCI SNR, a noise filtering was applied using its temporal repetitivity of $10 \mathrm{~min}$. The fusion process consists in increasing the FCI spatial resolution throughout the day thanks to the OLCI image.

In this manuscript, we firstly detail the image simulation process and the temporal filtering (section 2). Then we present our SSTF fusion method and the STARFM fusion method in order to compare their results (section 3). In this section, we also propose a validation methodology. In the following section, we present image simulation results and the comparison of the two fusion methods performances (section 4). Finally, we discuss the results (section 5).

\section{Image simulation and first processing}

As OLCI and FCI have not yet been launched, the images need to be simulated. The reference images were also required to be simulated for the purpose of fusion validation with the spatial resolutions of OLCI and the spectral and temporal resolutions of FCI. The image simulations are performed in four steps.

First, dynamic maps of $C h l$ and SPM are generated using hydro-biogeochemical and hydrosedimentary models. Then the corresponding Inherent Optical Properties (IOPs) are computed and used as inputs for a water radiative transfer model to produce remote sensing reflectance images. The atmospheric radiative transfer is eventually modeled to produce Top Of Atmosphere radiance. The spectral bands, the spatial resolution and the SNR of each sensor are considered for the image simulation. Figure 1 presents the flowchart of the image simulation process. 
[insert Figure 1]

Some pre-processing, such as temporal noise filtering for the FCI images and the atmospheric correction for both images, is also applied to the simulated images before the fusion process.

\subsection{Period and area of simulation}

OLCI and FCI images are simulated on the eastern gulf of Lion located on the Mediterranean coast of France. This area is highly representative of the applications of interest because it houses the Rhone River mouth and the urban outfall (Cortiou) of the second most populous town in France (Marseille). The images are simulated for May $18^{\text {th }}$ and $19^{\text {th }} 2008$ because these 2 days correspond to different dynamics. The first one is a high dynamic day, due to the rainfall, compared to the second one.

\subsection{Temporal seascape modelling}

The temporal variability of the water composition (here $C h l$ and $S P M$ ) is obtained by models. The hydrodynamic ocean model MARS3D (3D hydrodynamic Model for Application at Regional Scale, IFREMER) [21]. The input data of this model are: the water river contribution, the liquid input from the Waste water Treatment Plant contributions, meteorological parameters (pressure, wind speed and direction, air temperature, rainfall, heat fluxes).

MARS3D was also coupled with a numerical Hydro-Sedimentary model (hereafter called MS model) described in [22] in order to model the sediment dynamics in the Gulf of Lion, taking into account the liquid and solid outflow from the Rhone River, the coastal rivers and the Cortiou outfall. All details concerning the model processes, input and output can be found in the bibliography [22]-[24]. This model provides hourly Suspended Particle Matter (SPM) maps at each model grid cell, with $400 \mathrm{~m}$ spatial resolution and 30 vertical levels (3D model).

MARS3D was also coupled with the ECO3M model (Ecological Modular Mechanistic Model) [25], designed to model the biogeochemical carbon, nitrogen and phosphorus cycles of aquatic ecosystems and validated for the years 2007 and 2008 [24], [26]. The input data of this model are, in addition to those for hydrodynamics: the rivers and Waste water Treatment Plant biogeochemical contributions (nutrients, organic matter), the nutrient inputs from the atmosphere, and biogeochemical conditions on the Gulf of Lion [26]. This model provides $C h l$ and Particulate Organic Carbon (POC) maps every 10 minutes with $400 \mathrm{~m}$ spatial resolution in 3 dimensions (with 30 vertical levels).

The 3D water compositions maps of $C h l, P O C$ and inorganic SPMnorg were converted into 2D maps by integration of concentration along a vertical profile, [27]-[29] show that the influence of the vertical profile of the water compositions on the water surface reflectance can be described by a decreasing exponential function depending on depth. The slope of this exponential function depends on the light attenuation coefficient $K_{d}$. The influence of the vertical profile decreases with the turbidity at the surface level. 
Because the next step requires $S P M$ maps, we needed to obtain Suspended Organic Matter ( $S P M_{\text {org }}$ ) maps that can be deduced from $P O C$ maps [30]:

$$
S P M_{\text {org }}=2.6 P O C
$$

The $S P M_{n o r g}$ is directly obtained from the MARS3D Sediment-Transport model and the $S P M$ is then obtained by the sum of organic and non-organic SPM:

$$
S P M=S P M o r g+S P M n o r g
$$

Because no available model provides the CDOM 3D maps, we deduced these maps from the SPM maps with the relation $a_{y}(442) / S P M=0.0633$ found between MERIS products and confirmed by [31].

Because the original seascape maps (Chl, SPM and $C D O M$ ) have a $400 \mathrm{~m}$ resolution and we need to simulate at best $300 \mathrm{~m}$ resolution images. The maps were then over-sampled in order to reach the best resolution of the 2 sensors $(300 \mathrm{~m})$ using the cubic convolution technics. These $300 \mathrm{~m}$ resolution maps were then used as inputs of the image simulation chain.

\subsection{Sea surface reflectance modeling}

The Inherent Optical Properties (IOPs) were computed using the Chl, SPM and CDOM input maps considering the total absorption coefficient $a$, the total scattering coefficient $b$ and the backscattering coefficient $b_{b}$ as the sum of the different water components IOP. The water components are given from [28], [30]-[34].

Hydrolight [32] is a water radiative transfer model that enables the remote sensing reflectance images at surface level to be computed according to the IOPs. As computing Hydrolight for each pixel is too time consuming, look-up tables (LUTS) of remote sensing reflectance $R_{r s}$ are generated using Hydrolight version 5 [33]. These LUTs are generated according to the IOPs such as the absorption coefficient $a$, particle scattering coefficients $b_{p}$, the direct surface reflectance $R_{\text {refl }}$ and also according to the wind speed $W_{s p d}$ and the angular geometry ( $\theta_{t}$ the sensor zenith angle, $\Delta_{\phi}$ the relative azimuth angle and $\theta_{s}$ the sun zenithal angle).

\subsection{Atmospheric transfer modeling}

Because the sensor SNR are given for a specific Top Of Atmosphere radiance (TOA), the atmospheric transfer needs to be simulated. The atmosphere is simulated using the atmospheric model MODTRAN (MODerate resolution TRANsmittance and radiance code) which is one of the most used models for atmospheric radiative transfer by the remote sensing community [35], [36].

The TOA radiance was computed according to the four-stream radiative transfer theory. It results from 3 contributions: the atmospheric reflection, the direct target reflection and the surface 
environmental reflection. The radiance is given by the relationship (3), depending on the surface reflectance and variables described below.

$$
L_{T O A}=\frac{E_{s} \mu_{s}}{\pi}\left[\rho_{a t m}+\frac{t_{s}}{1-\rho_{e} S}\left(t_{d i r} \rho+t_{d i f} \rho_{e}\right)\right]
$$

where $E_{S}$ is the extraterrestrial solar radiance, $\rho_{A t m}$ the atmosphere intrinsic reflectance (molecular and aerosol), $\rho$ the target surface reflectance, $\rho_{e}$ the environmental surface reflectance, $t_{S}$ the total downwelling transmittance from the sun target, $t_{\text {dif }}$ the diffuse upwelling transmittance, $\mathrm{t}_{\text {dir }}$ the direct upwelling transmittance, $\mu_{S}$ the sun zenith angle cosine and $S$ the atmosphere spherical reflectance. $E_{s}, \rho_{A t m}, S, t_{s}, t_{d i r}, t_{d i f}$ are spectrally dependent and are given by the MODTRAN model. In this study, we chose the maritime aerosol profile proposed by MODTRAN because the visibility and relative humidity of this model are the closest to the local atmospheric conditions.

\subsection{Sensor simulation}

Regarding the spectral resolution, the remote sensing reflectance is integrated over the spectral bands corresponding to each sensor. Because the water color signal can be neglected after $780 \mathrm{~nm}$ [34] we simulated only the first 12 spectral bands of OLCI and the first 3 spectral bands of FCI (Table 1). The spectral response is given for the 2 sensors Figure 2(a) and Figure 2(b).

[insert Table 1]

[insert Figure 2]

Regarding the spatial resolution, the OLCI TOA image already has the right resolution (300 $\mathrm{m})$ but the FCI TOA image needs to be down-sampled in order to reach the right FCI resolution $(1 \mathrm{~km})$. The down-sampling is applied to the radiance images with the pixel aggregate method (filtering and subsampling).

Regarding the temporal resolution, FCI images are simulated every 10 minutes while OLCI images are simulated once a day at 10:00 am. For the latitude of Marseille (43.3 ${ }^{\circ}$ ), OLCI revisit period will be around 1 day once Sentinel 3A and 3B will be launched [38].

Concerning the SNR, the main sources of noise for the sensor are divided into 3 main categories: the photonic noise, the read noise and the quantization noise. The two last noises can be neglected thanks to the high quality of the electronic components and to the high quantization resolution of OLCI and FCI (12 bits). The photonic noise is the most significant. It is proportional to the number of photons captured. The flux of photons received is subject to a Poisson process, and therefore the noise is proportional to the signal square root (4). This noise is considered to be Gaussian, with a standard deviation $\left(\sigma_{\mathrm{ph}}\right)$. 


$$
\sigma_{\mathrm{ph}}=\sqrt{\alpha_{p h} \times L_{T O A}}
$$

The Signal to Noise Ratio (SNR) represents the magnitude of all the noises regarding the signal. This ratio depends on the amplitude of the signal and can also vary with the wavelength. The total noise can be considered as a white Gaussian noise with a standard deviation $\sigma_{b}(5)$.

$$
\sigma_{b}=\sqrt{\alpha_{p h} \times L_{\mathrm{TOA}}}=\frac{\sqrt{L_{T O A} \times L_{\mathrm{ref}}}}{S N R}
$$

$L_{r e f}$ and SNR were given by Thales Alenia Space, depending on the spectral bands. This noise is added on the Top Of Atmosphere radiance images.

For a given channel, the noise level varies with the square root of Top Of Atmosphere radiance, knowing that Lref/SNR ratio is unchanged for a given channel. FCI sensor has a SNR in line with the requirements of atmospherics measurements whereas OLCI is dimensioned for ocean color applications which requires higher SNR.

\subsection{First processing}

\section{Filtering}

Because FCI noise level is not compatible with an accurate estimation of the concentration of Chl, $S P M$ or $C D O M$ [8] and because its temporal frequency of one image every 10 minutes is over dimensioned for the water color monitoring, we decided to increase the FCI SNR thanks to its high temporal repetitivity. Five consecutive FCI images are then averaged in order to obtain a filtered image every hour with an improved SNR, more suitable to coastal applications. The standard deviation of the remaining noise is then reduced by a factor of 3 .

\section{Inversion of the atmospheric transfer}

Because the fusion process needs to be applied on reflectance images (normalized from acquisition conditions), an atmospheric correction is computed. To study the error due only to the fusion process, the atmospheric correction is considered to be perfect. As a consequence, a simple inversion of the atmospheric model has been performed (6) with the parameters previously defined (3).

$$
\rho=\left[\frac{L_{T O A} \times \pi}{E_{S} \times \mu_{S}}-\rho_{a t m}-\frac{t_{S} \times t_{d i r} \times \rho_{e}}{1-S \times \rho_{e}}\right] \times\left(\frac{1-S \times \rho_{e}}{t_{S} \times t_{d i r}}\right)
$$




\section{Methodology}

\subsection{Fusion methods}

\section{$\underline{\text { Spatial Spectral Temporal Fusion (SSTF) method }}$}

Our fusion process consists in adding the OLCI spatial details into the 3 FCI spectral bands. The resulting image has thus, the spatial resolution of OLCI and the spectral and temporal resolutions of FCI. The SSTF method is inspired by the method proposed by Q. Vanhellemont [20] who combined data from the single broad red band of the SEVIRI GEO sensor with the corresponding band of the MODIS sun-synchronous sensor. This method considers that the temporal variations of the reflectance measured by two different sensors for a same interval are equals. Using this method, which has been adapted to our sensors, the ratio between 2 consecutive FCI images is multiplied by the high spatial resolution OLCI image with the corresponding band (7).

$$
\operatorname{OLCI}\left(\lambda_{i}, t_{1}\right)=\frac{F C I\left(\lambda_{i}, t_{1}\right)}{F C I\left(\lambda_{i}, t_{0}\right)} \times \operatorname{OLCI}\left(\lambda_{i}, t_{0}\right)
$$

The multispectral bands of the two sensors were associated according to their spectral proximity. FCI $1^{\text {st }}$ band $(444 \mathrm{~nm})$ is fused with the OLCI $3^{\text {rd }}$ band $(442 \mathrm{~nm})$, FCI $2^{\text {nd }}$ band $(510 \mathrm{~nm})$ with OLCI $5^{\text {th }}$ band $(510 \mathrm{~nm})$ and FCI $3^{\text {rd }}$ band $(640 \mathrm{~nm})$ with OLCI $7^{\text {th }}$ band $(620 \mathrm{~nm})$.

To implement this method, the FCI images need to be oversampled to reach the OLCI spatial resolution. To oversample the FCI images, two methods were compared: the cubic convolution [39] and the B-spline [40] interpolation methods .

Only one OLCI image is acquired per day but the resulting fusion product $\left(\mathrm{t}_{\mathrm{i}-1}\right)$ is used to perform the next fusion with the existing FCI $\left(\mathrm{t}_{\mathrm{i}}\right)$ and to generate a new product $\left(\mathrm{t}_{\mathrm{i}}\right)$. This method was applied throughout 2 consecutive days.

This method was compared to the STARFM existing method.

\section{$\underline{\text { STARFM }}$}

STARFM method (Spatial and Temporal Adaptive Reflectance Fusion Model) [19] is also a multispectral-spatial-temporal fusion model but the predicted image is computed differently. The high resolution predicted image $\operatorname{OLCI}\left(\mathrm{t}_{0}\right)$, is considered equal to the oversampled coarse resolution image FCI $\left(\mathrm{t}_{0}\right)$, added to the difference between the 2 sensors images at the acquisition time. A selection of neighboring pixels within a moving window is operated on the OLCI image according to the spectral similarity with the central pixel. The selected pixels are then weighted $\left(\mathrm{W}_{\mathrm{ijk}}\right)$ according to the spectral, spatial and temporal distances between the central and the selected pixels (8). 


$$
\begin{array}{r}
\operatorname{OLCI}\left(x_{w / 2}, y_{w / 2}, t_{0}\right) \\
=\sum_{w}^{w} \sum_{i=1}^{w} \sum_{j=1}^{n} W_{i j k} \times\left(F C I\left(x_{i}, y_{i}, t_{0}\right)\right. \\
\left.+\operatorname{OLCI}\left(x_{i}, y_{i}, t_{k}\right)-\operatorname{FCI}\left(x_{i}, y_{i}, t_{k}\right)\right)
\end{array}
$$

where $w$ is the searching window size, $i$ and $j$ the spatial location in the image, $t_{0}$ and $t_{k}$ respectively the prediction and the acquisition date of FCI and OLCI images. More details are available in [19]. This fusion method has never been applied to the fusion of GEO and LEO images before.

\subsection{Validation method}

\section{With the reference images}

The fusion products of the 2 methods can be compared to the reference images, having the same features (300 m, 3 bands, 10 min). The statistical index called ERGAS for "Relative Adimensional Global Error in Synthesis" (9) is used to assess the global quality of the fused images with more robustness than the RASE (Relative Average Spectral Error), regardless of the spatial resolution and the spectral bands. It also respects calibration and changes of units. The ideal value is 0 in case of perfect fusion, but its value remains correct under 3 [41].

$$
\operatorname{ERGAS}(A, B)=100 \times \frac{h}{l} \sqrt{\frac{1}{n b} \sum_{\lambda=1}^{n_{b}} \frac{\operatorname{RMSE}(A, B, \lambda)^{2}}{\operatorname{mean}(A, B, \lambda)^{2}}}
$$

where $h / l$ is the ratio between OLCI and FCI spatial resolutions and $n b$ is the number of fused bands.

We also measured the persistence which consists in calculating the ERGAS index between the reference images at each hour and the only OLCI image of the day. It shows the error due to the seascape variations in order to judge whether the fusion is useful or not. If the persistence error is lower than the fusion error, the fusion is pointless.

\section{$\underline{\text { With water composition maps }}$}

Radiometric indexes are good indicators to assess the fusion performance but sometimes they are not sufficient if the final application is not satisfied at the end of the study. The fusion methods can effectively be compared and evaluated on the accuracy of the water components estimation which is the final application of our fusion. This comparison can easily be made with the maps used as simulation input, which are the perfect "ground truth". 
Because the fusion product has only 3 bands, we cannot use an algorithm like OC5 which needs specific bands [42], so we chose to inverse a physical model developed by Lee [43],[44]. Lee's model inputs are $C h l, S P M$ and $C D O M$ concentrations. The IOP (absorption $a$, backscattering $b_{b}$ ) are derived from these inputs. $a$ and $b_{b}$ are additives, so they result from the sum of the pure seawater $a_{w}$, the phytoplankton coefficient $a_{\phi}$, the coefficient of Coloured Dissolved Organic Matter (CDOM) $a_{y}$, the non-algal particle coefficient $a_{n a p}$, the pure seawater scattering coefficient $b_{w}$, and the water particle scattering coefficient $b_{p}$. The second step provides the remote sensing reflectance, i.e. apparent optical properties (AOP), derived from the IOP and from the observation geometry.

This model can be reversed to estimate $C h l^{\prime}, S P M^{\prime}$ and $C D O M^{\prime}$ concentrations from the fusion product ( 3 bands, $300 \mathrm{~m}$ ), given in reflectance after atmospheric correction. The reverse model consists in varying the input concentrations in order to minimize the error between the model spectrum and the measured one. It requires the determination of the initial values and the range of variations for each concentration. An example is represented in Figure 3 for a Case 1 waters, with the remote sensing reflectance $\mathrm{R}_{\mathrm{rs}}$ corresponding to the initial values $\left(C h l=1 \mathrm{mg} . \mathrm{m}^{-3}, S P M=1 \mathrm{~g} . \mathrm{m}^{-}\right.$ ${ }^{3}$ and $C D O M=0.1 \mathrm{~m}^{-1}$ ) and $\mathrm{R}_{\mathrm{rs}}$ once the iterative process of optimization is over, i.e. $C h l=0.2 \mathrm{mg} \cdot \mathrm{m}^{-}$ , $S P M=0.01 \mathrm{~g} . \mathrm{m}^{-3}$ and $C D O M=0.01 \mathrm{~m}^{-1}$.

\section{[insert Figure 3]}

This method enables these three parameters to be determined simultaneously by using the remote sensing reflectance from at least three spectral bands between 400 and $800 \mathrm{~nm}$.

The relative errors are then computed between the input maps (Chl, SPM and CDOM) and the estimation maps (Chl', SPM', CDOM') from the images resulting from the fusion. The flowchart of fusion and validation process is presented Figure 4.

[insert Figure 4]

\section{Results}

\subsection{Results of simulation}

Figure 5 shows a color composite of the FCI simulated images (444, 510 and $640 \mathrm{~nm})$ on the Gulf of Lion at 2:00 pm without noise simulation (a), with noise simulation (b) and after noise filtering (c) and the corresponding horizontal profiles for the 3 bands (d), (e) and (f) corresponding to the red line on the images. On (d) we see the relevant information (the water color signal) while on (e) this information is combined with the noise. On (f), we can see that the noise is reduced compared to (e) and the information is preserved compared to (d). Figure 6 shows a color composite of the OLCI simulated image (442, 510 and $620 \mathrm{~nm}$ ) at 10:00 am without noise simulation (a) and with noise simulation (b). On OLCI images, the noise is so low that it is not visible on the image (b) but it can however be visualized on the profile (d). 
[insert Figure 5]

[insert Figure 6]

Spatial differences between images of FCI $(1 \mathrm{~km})$ and OLCI $(300 \mathrm{~m})$ can be noticed between Figure 5 and Figure 6.

\subsection{Results of fusion}

Figure 7 shows a color composite $(444,510$ and $640 \mathrm{~nm})$ of the fusion product with the SSTF method corresponding to the increase of FCI spatial resolution $(1 \mathrm{~km})$ thanks to the OLCI one (300 $\mathrm{m}$ ), without noise simulation (a), with noise simulation (b) and with FCI noise filtering (c). The spatial resolution was improved for the 3 images but the noise is very noticeable on (b). However, the fusion applied to FCI filtered images gives much better results (c) compared to (b).

[insert Figure 7]

\subsection{Result of validation}

\section{$\underline{\text { With reference images }}$}

In order to quantify accurately the error induced by the fusion process, the ERGAS statistical index was computed each hour between the fusion product and the simulated reference image.

The 2 oversampling methods enabling FCI images to reach OLCI spatial resolution, cubic convolution and B-spline ( $6^{\text {th }}$ degree), were compared and the B-spline method always provided an ERGAS value 1.5 times higher than those obtained with the cubic convolution method. Furthermore, the high reflectance values due to the high concentration of SPM on the shore created spatial fluctuations of reflectance (artefacts) that were still present in the resulting fused images. We then chose to use the cubic convolution resampling method in the fusion process.

On Figure 8, ERGAS index corresponding to May $18^{\text {th }}$ and $19^{\text {th }} 2008$ is computed between the fusion product and the reference images without noise. The ERGAS value corresponding to the persistence is also plotted. The ERGAS value being lower than the persistence proves that the fusion product provides more extensive information than the OLCI image alone and it then proves the usefulness of the fusion. But in case of significant seascape variations, the fusion can generate serious errors that are propagated to the next hour's fusion and cumulated throughout the day. Figure 8 shows that these errors are totally different from one day to the next. For example, with the images without noise, the average ERGAS is 0.9 for the first day while it is only 0.29 for the second day. During the first day with high seascape dynamics, the difference between fusion ERGAS and the persistence is much higher than for the second day. The fusion is still useful but the increase of seascape change induces an increase of the ERGAS error.

[insert Figure 8] 
To analyze the impact of the noise on the fusion performance, Figure 9 shows the evolution of the ERGAS index on May $18^{\text {th }} 2008$ with noiseless, noisy and FCI filtered input images, the persistence is plotted in red. The error of the fusion process for the first hour's fusion is around 0.3 with noiseless images, 0.9 with noisy images and 0.5 with filtered images as input. Then the error added from one hour to the next is only 0.1 whatever the input images. These values show that the noise reduction computed on the noisy FCI images has greatly improved the fusion product. The plot also shows that the error seems to be constant throughout the day. The persistence shows that the fusion provides more consistent information, even with noisy FCI images, than with the same OLCI image used throughout the day.

[insert Figure 9]

In order to visualize the spatial distribution of the accumulated errors at the end of the day, error maps between the reference and the final fusion images at 5:00 pm were displayed (Figure 10). On (a) we can see that the errors correspond to the plume variations (on the borders). On (b) and (c) the errors are spatially constant, due to the GEO sensor noise and they were reduced by $50 \%$ with the temporal FCI filtering (c).

[insert Figure 10]

$\underline{\text { With water composition maps }}$

We compared the water composition maps obtained after the fusion process with the input maps. On Figure 11, the mean relative errors of the estimated Chl (a), SPM (b) and CDOM maps (c) without noise during May 18th and 19th 2008 is plotted. The Chl is misestimated for the 2 days with relative errors around $64 \%$ and $60 \%, S P M$ is accurately estimated with $6.5 \%$ and $5.5 \%$ as the CDOM around 5.5 and $9 \%$.

We also plotted the errors on $C h l$ (a), SPM (b) and CDOM maps (c) with input images without noise, noisy and filtered for May 18th 2008 (Figure 12). The results are greatly improved by the temporal filtering of the FCI images. Without filtering, average relative errors on $C h l, S P M$ and CDOM estimation are respectively $258 \%, 16 \%$ and $28 \%$, whereas with filtering, they become $82 \%$, $8 \%$ and $12 \%$.

[insert Figure 11]

[insert Figure 12]

\subsection{Comparison with results from the STARFM algorithm}

The SSTF method was also compared with the existing STARFM method by computing the ERGAS index and relative errors on water component estimation during the day 18/05/2008 (Table 2). Using images filtered from the noise as input, average ERGAS is 1 with the SSTF method and 1.47 with the STARFM method. While SSTF relative errors are constant through the day, STARFM ones increase. Concerning the water component estimation, relative errors on Chl, SPM and $C D O M$ are respectively $79.9 \%, 8.87 \%$ and $13.9 \%$ with the SSTF method, and $63.1 \%, 8.52 \%$ 
and $13.85 \%$ with the STARFM method. The results are quite similar for SPM and CDOM but $c h l$ is really improved when estimated with STARFM fused images.

[insert Table 2]

\section{Discussion}

This work has shown that the fusion of LEO and GEO images has 2 sources of errors. The first is due to the seascape dynamics that increase the error between the fusion products and the images that would have been acquired by a sensor with the spatial resolution of the LEO sensor and the spectral and the temporal resolution of the GEO one. Because only one high spatial resolution image is acquired during the day, an error of propagation is measured by the ERGAS index throughout the day. The fusion was applied to noiseless simulated images and the spatial analysis showed that this radiometric error is located on the area of most variations, on the border of the plume for example (Figure 10). But whatever the seascape dynamics, the persistence study showed that the fusion is always useful (ERGAS with persistence higher than ERGAS with fusion, Figure 8). The seascape variations during the day also have a noticeable impact on the estimation maps. The relative errors on $S P M$ and the $C D O M$ estimation are low (respectively $\sim 5.5 \%$ and $\sim 9 \%$ ) and increase slightly throughout the day. Chl is misestimated ( $60 \%)$ because SPM obscures the influence of $\mathrm{Chl}$ on the water surface reflectance, and remains constant (Figure 11).

The second source of errors is due to the SNR of FCI images. Without noise filtering the ERGAS value is around 1, Chl is misestimated by $250 \%, S P M$ by $16 \%$ and CDOM by $28 \%$. The error is then acceptable for $S P M$ and $C D O M$ values even without noisy images but not for $C h l$.

When FCI images are temporally filtered, the ERGAS value is improved and approaches the fusion without noise (0.75/0.6), proving that the temporal filtering is really effective (Figure 9). On the water component estimation, filtering is much more effective because all components are much more accurately estimated: chl (250/80\%), SPM (16/8\%) and CDOM (28/12\%) (Figure 12).

If we compare the 2 sources of errors, we can say that without temporal filtering, the noise impact is higher than the seascape dynamics on the fusion performance but when the temporal filtering is applied, the seascape dynamics becomes the main source of error.

The remaining noise could also be reduced by using an adaptive temporal filtering. We tried to temporally filter the images with the median filter but the resulting SNR of the filtered images was lower than the one obtained by temporal average filtering. On the contrary, the seascape dynamics could not be reduced because this is useful information that should be kept and analyzed.

Compared to other fusion methods like MMT or CNMF, this method doesn't make the assumption that the landscape has not changed between the 2 acquisitions and this is important in marine areas. Even if the fusion error increases with the seascape change, the information concerning change is kept and used by this method. Furthermore, our method was compared to the STARFM method, never tested on LEO and GEO images before. SSTF provides a lower ERGAS index than STARFM but results on water component estimation are quite similar except for $c h l$ having lower error with 
STARFM. Compared to the STARFM method, no weighting function in a searching window is needed, SSTF is then less time consuming than STARFM.

Concerning the misestimation of $\mathrm{Chl}$ after the fusion between FCI and OLCI images, we can surmise that in Case 1 waters, the estimation of $C h l$ would be better because the influence of $C h l$ on reflectance would not be masked by the SPM presence and the estimation would then be more reliable.

One limitation of the SSTF method is that our fusion process does not provide the 12 bands of OLCI sensor. The original idea was to provide an image combining the best qualities of the 2 sensors (spatial, spectral and temporal features). We combined the spatial resolution of OLCI and the temporal resolution of FCI but we have not yet succeeded in obtaining fused images with OLCI spectral resolution. Our idea was to use a physical model of reflectance to add a spectral fusion phase after the spatial one (presented here), but this process would not have added more information to the resulting image. We therefore disregarded this idea for the moment. The improvement of the SNR and the spatial resolution of the FCI images are already a notable progress. But to estimate $C h l$ concentrations, more than 3 bands would certainly be helpful.

Concerning the future applications of these fusion products, 3 main directions can be cited. The first one is the monitoring of daily biological dynamics in offshore waters (Case 1 waters). Monitoring the Chlorophyll enables the phytoplankton cycle to be studied, contributor to photosynthetic activity and contributes indirectly to monitoring the carbon cycle. In a preliminary study of FCI and OLCI sensors for the estimation of water composition, we proved that for Case 1 waters, the $C h l$ estimation is possible with low errors with the $3 \mathrm{FCI}$ broad bands after a noise filtering. The second application would be the monitoring of coastal area dynamics, to follow the river mouth and the river plume as we did in this paper for example.

Finally, the last application of this fusion method will be the increase of the spatial coverage to take into account the cloud cover over some overcast areas. The advantage of GEO sensor is to provide several images during the day ( $60 \mathrm{FCI}$ images for example). This number of images would make available limited area without cloud each day. These local images could be fused with the last LEO sensor image received. The image could then be recomposed from different local fusion products. Having a composite product with no clouds is one of the aims of the color water community (see IOCCG reports), but this product will have to be used very carefully because of the temporal inconsistency of concomitant pixels which could have been acquired at different times. 


\section{Conclusion}

This work has shown that even with a lower SNR, GEO images can provide useful information in addition to LEO ocean color sensors even for ocean color surveys because the temporal resolution can balance the low SNR by using temporal filtering. A fusion method which was given the name SSTF was then proposed to combine the spatial resolution of the LEO sensor with the temporal resolution of the GEO sensor in order to obtain multispectral GEO images enabling a quasicontinuous monitoring of water composition. The SSTF method was applied to the future FCI (MTG) and OLCI (Sentinel-3) images that were simulated to assess the fusion method. Because FCI is dimensioned for meteorological applications and not for ocean observations, a noise filtering was applied and enabled the noise to be reduced by $39 \%$ on the 3 bands. The SSTF method was compared with STARFM. The results are quite similar with both methods but we noticed that SSTF gives better results in case of high landscape dynamic while STARFM is more efficient with noisy images.

\section{Acknowledgment}

The authors thank the French region Provence-Alpes-Côte d'Azur and Thales Alenia Space for their financial support. The authors are grateful to the European Space Agency for the funding of MTG and Sentinel 3 programs from which the simulation are derived.

\section{References}

[1] T. Platt, "Why Ocean Colour? The Societal Benefits of Ocean-Colour Technology," IOCCG, Dartmouth, Canada, Rep. 7, 2008.

[2] W. Hovis, D. Clark, F. Anderson, R. Austin, W. Wilson, E. Baker, D. Ball, H. Gordon, J. Mueller, S. El-Sayed, and others, "Nimbus-7 Coastal Zone Color Scanner: system description and initial imagery," Science, vol. 210, no. 4465, pp. 60-63, 1980.

[3] S. B. Hooker, E. R. Firestone, W. E. Esaias, G. C. Feldman, W. W. Gregg, and C. R. Mcclain, "SeaWiFS technical report series. Volume 1: An overview of SeaWiFS and ocean color," 1992.

[4] W. E. Esaias, M. R. Abbott, I. Barton, O. B. Brown, J. W. Campbell, K. L. Carder, D. K. Clark, R. H. Evans, F. E. Hoge, H. R. Gordon, and others, "An overview of MODIS capabilities for ocean science observations," Geosci. Remote Sens. IEEE Trans. On, vol. 36, no. 4, pp. 1250-1265, 1998.

[5] G. Zibordi, F. Mélin, J.-F. Berthon, and E. Canuti, "Assessment of MERIS ocean color data products for European seas," Ocean Sci., vol. 9, no. 3, pp. 521-533, 2013.

[6] S. Hlaing, T. Harmel, A. Gilerson, R. Foster, A. Weidemann, R. Arnone, M. Wang, and S. Ahmed, "Evaluation of the VIIRS ocean color monitoring performance in coastal regions," Remote Sens. Environ., vol. 139, pp. 398-414, 2013. 
[7] J. Nieke, C. Mavrocordatos, C. Donlon, B. Berruti, T. Garnier, J.-B. Riti, and Y. Delclaud, Ocean and Land Color Imager on Sentinel-3 in Optical Payloads for Space Missions, John Wiley \& Sons, Ltd. Chichester, UK: S.-E. Qian, 2015.

[8] K. Ruddick, G. Neukermans, Q. Vanhellemont, and D. Jolivet, "Challenges and opportunities for geostationary ocean colour remote sensing of regional seas: A review of recent results," Remote Sens. Environ., vol. 146, pp. 63-76, 2014.

[9] J.-K. Choi, Y. J. Park, J. H. Ahn, H.-S. Lim, J. Eom, and J.-H. Ryu, "GOCI, the world's first geostationary ocean color observation satellite, for the monitoring of temporal variability in coastal water turbidity," J. Geophys. Res. Oceans, vol. 117, no. C9, 2012.

[10] G. Neukermans, B. Nechad, and K. Ruddick, "Optical remote sensing of coastal waters from geostationary platforms: a feasibility study-mapping total suspended matter with SEVIRI," in Proceedings of the XIX Ocean Optics Conference, 2008.

[11] G. Neukermans, K. G. Ruddick, and N. Greenwood, "Diurnal variability of turbidity and light attenuation in the southern North Sea from the SEVIRI geostationary sensor," Remote Sens. Environ., vol. 124, pp. 564-580, 2012.

[12] F. Faure, P. Coste, and G. Kang, "The GOCI instrument on COMS mission-The first geostationary ocean color imager," in Proceedings of the International Conference on Space Optics (ICSO), 2008, pp. 14-17.

[13] T. Ranchin, B. Aiazzi, L. Alparone, S. Baronti, and L. Wald, "Image fusion-the ARSIS concept and some successful implementation schemes," ISPRS J. Photogramm. Remote Sens., vol. 58, no. 1-2, pp. 4-18, 2003.

[14] B. Zhukov, D. Oertel, F. Lanzl, and G. Reinhackel, "Unmixing-based multisensor multiresolution image fusion," IEEE Trans. Geosci. Remote Sens., vol. 37, no. 3, pp. 12121226, 1999.

[15] A. Minghelli-Roman, M. Mangolini, M. Petit, and L. Polidori, "Spatial resolution improvement of MeRIS images by fusion with TM images," IEEE Geosci. Remote Sens., vol. 39, no. 7, pp. 1533-1536, 2001.

[16] A. Minghelli-Roman, L. Polidori, S. Mathieu-Blanc, L. Loubersac, and F. Cauneau, "Spatial Resolution Improvement by Merging MERIS-ETM Images for Coastal Water Monitoring," IEEE Geosci. Remote Sens. Lett., vol. 3, no. 2, pp. 227-231, 2006.

[17] N. Yokoya, T. Yairi, and A. Iwasaki, "Hyperspectral, multispectral, and panchromatic data fusion based on coupled non-negative matrix factorization," in Hyperspectral Image and Signal Processing: Evolution in Remote Sensing (WHISPERS), 2011 3rd Workshop on, Lisbon, 2011, pp. 1-4.

[18] D. Sylla, A. Minghelli-Roman, P. Blanc, A. Mangin, F. d'Andon, and O. Hembise, "Fusion of multispectral images by extension of the pan-sharpening ARSIS method," IEEE J. Sel. Top. Appl. Earth Obs. Remote Sens., vol. 7, no. 5, pp. 1781-1791, 2014.

[19] Feng Gao, J. Masek, M. Schwaller, and F. Hall, "On the blending of the Landsat and MODIS surface reflectance: predicting daily Landsat surface reflectance," IEEE Trans. Geosci. Remote Sens., vol. 44, no. 8, pp. 2207-2218, 2006.

[20] Q. Vanhellemont, G. Neukermans, and K. Ruddick, "Synergy between polar-orbiting and geostationary sensors: Remote sensing of the ocean at high spatial and high temporal resolution," Remote Sens. Environ., vol. 146, pp. 49-62, 2014.

[21] P. Lazure and F. Dumas, "An external-internal mode coupling for a 3D hydrodynamical model for applications at regional scale (MARS)," Adv. Water Resour., vol. 31, no. 2, pp. 233-250, 2008. 
[22] R. Verney, C. Jany, B. Thouvenin, I. Pairaud, M. I. Vousdoukas, C. Pinazo, F. Ardhuin, and P. Cann, "Sediment transport in the Bay of Marseille: Role of extrem events," presented at the Coastal Dynamics 2013-7th International Conference on Coastal dynamics, Arcachon, France, 2013.

[23] I. L. Pairaud, J. Gatti, N. Bensoussan, R. Verney, and P. Garreau, "Hydrology and circulation in a coastal area off Marseille: Validation of a nested 3D model with observations," J. Mar. Syst., vol. 88, no. 1, pp. 20-33, 2011.

[24] M. Fraysse, C. Pinazo, V. M. Faure, R. Fuchs, P. Lazzari, P. Raimbault, and I. Pairaud, "Development of a 3D Coupled Physical-Biogeochemical Model for the Marseille Coastal Area (NW Mediterranean Sea): What Complexity Is Required in the Coastal Zone?," PLoS ONE, vol. 8, no. 12, p. e80012, 2013.

[25] M. Baklouti, V. Faure, L. Pawlowski, and A. Sciandra, "Investigation and sensitivity analysis of a mechanistic phytoplankton model implemented in a new modular numerical tool (Eco3M) dedicated to biogeochemical modelling," Prog. Oceanogr., vol. 71, no. 1, pp. 34$58,2006$.

[26] M. Fraysse, I. Pairaud, O. N. Ross, V. M. Faure, and C. Pinazo, "Intrusion of Rhone River diluted water into the Bay of Marseille: Generation processes and impacts on ecosystem functioning," J. Geophys. Res. Oceans, vol. 119, no. 10, pp. 6535-6556, 2014.

[27] M. Lei, A. Minghelli-Roman, A. Bricaud, and J. M. Froidefond, "Simulation of Future Geostationary Ocean Color Images," IEEE J. Sel. Top. Appl. Earth Obs. Remote Sens., vol. 5, pp. 173-182, 2011.

[28] H. Gordon and D. Clark, "Remote sensing optical properties of a stratified ocean: an improved interpretation," vol. 19, no. 20, pp. 3428-3430, 1980.

[29] J. R. Zaneveld, A. Barnard, and E. Boss, "Theoretical derivation of the depth average of remotely sensed optical parameters," Opt. Express, vol. 13, no. 22, pp. 9052-9061, 2005.

[30] M. Babin, A. Morel, V. Fournier-Sicre, F. Fell, and D. Stramski, "Light scattering properties of marine particles in coastal and open ocean waters as related to the particle mass concentration," Limnol Ocean, vol. 48, pp. 843-859, 2003.

[31] M. Babin, D. Stramski, G. M. Ferrari, H. Claustre, A. Bricaud, G. Obolensky, and N. Hoepffner, "Variations in the light absorption coefficients of phytoplankton, nonalgal particles, and dissolved organic matter in coastal waters around Europe," J. Geophys. Res., vol. 108, p. 3211, 2003.

[32] C. D. Mobley, Light and Water: Radiative Transfer in Natural Waters, Academic Press. 1994.

[33] A. Minghelli-roman, M. Lei, M. Fraysse, I. Pairaud, and C. Verney, "Image simulation of the geostationary sensor geoocapi for the water quality survey of a dynamic coastal area," presented at the IGARSS, 2014.

[34] R. Pope and E. Fry, “Absorption spectrum $(380-700 \mathrm{~nm})$ of pure water. II. Integrating cavity measurements," vol. 36, no. 33, pp. 8710-8723, 1997.

[35] A. Berk, L. S. Bernstein, G. P. Anderson, P. K. Acharya, D. C. Robertson, J. H. Chetwynd, and S. M. Adler-Golden, "MODTRAN cloud and multiple scattering upgrades with application to AVIRIS," Remote Sens. Environ., vol. 65, no. 3, pp. 367-375, 1998.

[36] W. Verhoef and H. Bach, "Simulation of hyperspectral and directional radiance images using coupled biophysical and atmospheric radiative transfer models," Remote Sens. Environ., vol. 87, no. 1, pp. 23-41, 2003.

[37] P. J. Burt and E. H. Adelson, "The Laplacian pyramid as a compact image code," IEEE Trans. Commun., vol. 31, no. 4, pp. 532-540, 1983. 
[38] K. Fletcher, "Sentinel-3: ESA's Global Land and Ocean Mission for GMES Operational Services," ESA communications, 2012.

[39] R. G. Keys, "Cubic convolution interpolation for digital image processing," IEEE Trans. Acoust. Speech Signal Process., vol. ASSP-29, no. 6, 1981.

[40] M. Unser, A. Aldroubi, and M. Eden, "B-spline signal processing. I. Theory," IEEE Trans. Signal Process., vol. 41, no. 2, pp. 821-833, 1993.

[41] L. Wald, "Quality of high resolution synthesised images: Is there a simple criterion?," in Third conference "Fusion of Earth data: merging point measurements, raster maps and remotely sensed images, " Sophia Antipolis, France, 2000, pp. 99-103.

[42] F. Gohin, J. N. Druon, and L. Lampert, "A five channel chlorophyll concentration algorithm applied to SeaWiFS data processed by SeaDAS in coastal waters," Int. J. Remote Sens., vol. 23, no. 8, pp. 1639-1661, 2002.

[43] Z. P. Lee, K. L. Carder, and R. A. Arnone, "Deriving inherent optical properties from water color: a multiband quasi-analytical algorithm for optically deep waters," Appl. Opt., vol. 41, no. 22, 2002.

[44] Z. P. Lee, K. L. Carder, C. D. Mobley, R. G. Steward, and J. S. Patch, "Hyperspectral remote sensing for shallow waters. I. A semianalytical model," Appl. Opt., vol. 37, no. 27, pp. 63296338, 1998.

Tables:

\begin{tabular}{|c|c|c|}
\hline $\begin{array}{c}\text { FCI } \\
\text { Channels }\end{array}$ & $\begin{array}{c}\text { Center } \\
\text { (nm) }\end{array}$ & $\begin{array}{c}\text { Width } \\
\text { (nm) }\end{array}$ \\
\hline 1 & 444 & 60 \\
\hline 2 & 510 & 40 \\
\hline 3 & 640 & 50 \\
\hline
\end{tabular}

(a)

\begin{tabular}{|c|c|c|}
\hline $\begin{array}{c}\text { OLCI } \\
\text { Channels }\end{array}$ & $\begin{array}{c}\text { Center } \\
(\mathrm{nm})\end{array}$ & $\begin{array}{c}\text { Width } \\
(\mathrm{nm})\end{array}$ \\
\hline 1 & 400 & 15 \\
\hline 2 & 412 & 10 \\
\hline 3 & 442 & 10 \\
\hline 4 & 490 & 10 \\
\hline 5 & 510 & 10 \\
\hline 6 & 560 & 10 \\
\hline 7 & 620 & 10 \\
\hline 8 & 665 & 10 \\
\hline 9 & 681 & 7.5 \\
\hline 10 & 709 & 10 \\
\hline 11 & 753 & 7.5 \\
\hline 12 & 761 & 2.5 \\
\hline
\end{tabular}

(b)

Table 1: FCI (a) and OLCI (b) channels. 


\begin{tabular}{|c|c|c|c|c|c|c|c|c|}
\hline & \multicolumn{2}{|c|}{$\begin{array}{c}\text { ERGAS with } \\
\text { reference images }\end{array}$} & \multicolumn{3}{c|}{ Relative errors with water composition maps (\%) } \\
\hline \multirow{2}{*}{ time } & SSTF & STARFM & \multicolumn{3}{c|}{ SSTF } & \multicolumn{3}{c|}{ STARFM } \\
\cline { 5 - 9 } & & & Chl & SPM & CDOM & Chl & SPM & CDOM \\
\hline 8:00 AM & 1.040 & 1.074 & 86.38 & 9.26 & 14.91 & 66.82 & 9.65 & 14.91 \\
\hline 9:00 AM & 0.709 & 0.647 & 79.64 & 8.15 & 12.79 & 64.38 & 7.93 & 12.47 \\
\hline 10:00 AM & 0.000 & 0.000 & 0.00 & 0.00 & 0.00 & 0.00 & 0.00 & 0.00 \\
\hline 11:00 AM & 0.629 & 0.701 & 77.49 & 8.24 & 12.77 & 60.77 & 6.15 & 10.17 \\
\hline 12:00 AM & 0.887 & 1.087 & 85.41 & 8.39 & 13.40 & 60.73 & 6.89 & 11.75 \\
\hline 1:00 PM & 1.077 & 1.390 & 83.08 & 8.53 & 13.69 & 59.79 & 7.49 & 13.09 \\
\hline 2:00 PM & 1.127 & 1.608 & 77.21 & 8.54 & 13.71 & 60.30 & 8.05 & 13.95 \\
\hline 3:00 PM & 1.139 & 1.722 & 76.66 & 8.84 & 13.85 & 60.38 & 8.72 & 14.62 \\
\hline 4:00 PM & 1.110 & 1.832 & 76.51 & 9.09 & 13.89 & 62.89 & 9.35 & 15.13 \\
\hline 5:00 PM & 1.106 & 1.912 & 77.20 & 9.38 & 14.29 & 65.54 & 9.97 & 15.80 \\
\hline 6:00 PM & 1.186 & 2.031 & 80.29 & 10.3 & 15.73 & 69.31 & 11.0 & 16.67 \\
\hline
\end{tabular}

Table 2: ERGAS index between the fusion products of the 2 fusion methods and the reference images and relative error between estimated water contents and input maps for the 18/5/2008.

Figures:

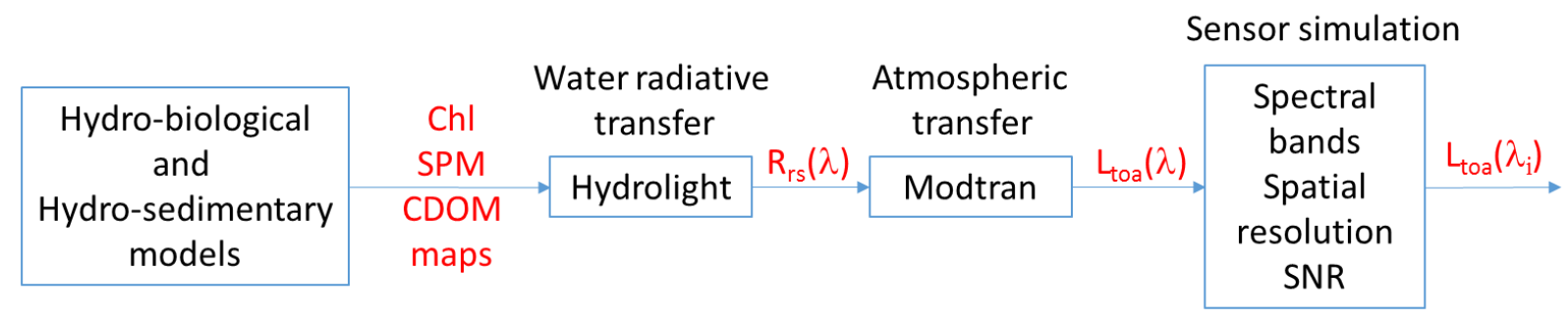

Figure 1: Flowchart of image simulation process. 


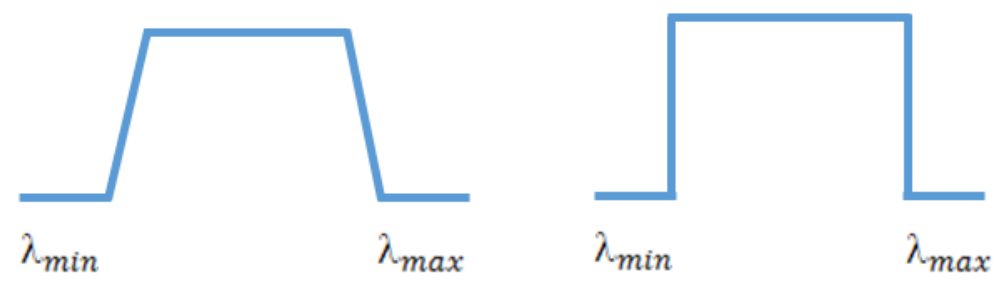

(a)

(b)

Figure 2: FCI (a) and OLCI (b) spectral responses.

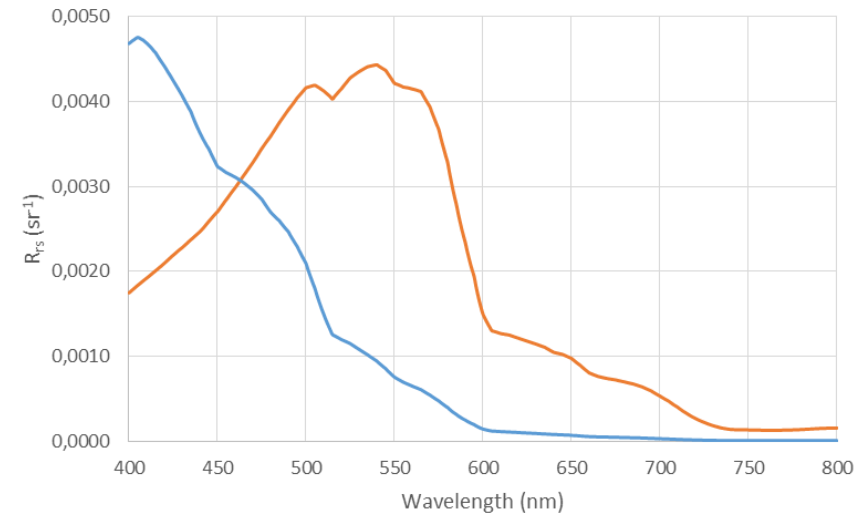

—Initial Rrs _ Final Rrs

Figure 3: Initial and final reflectance of the optimization process.

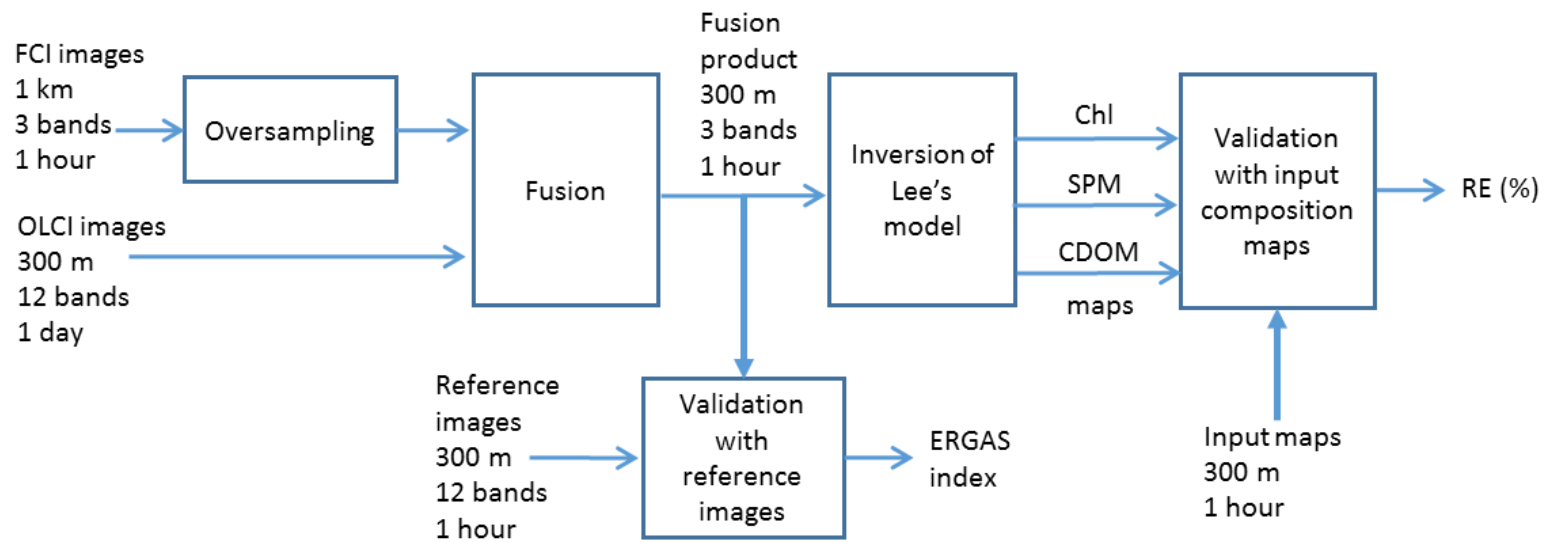

Figure 4: Flowchart of fusion and validation process. 


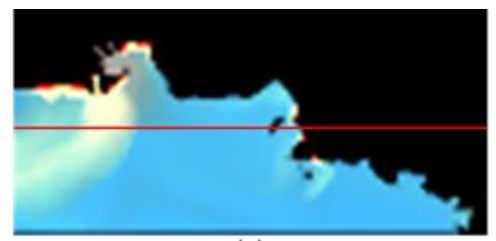

(a)

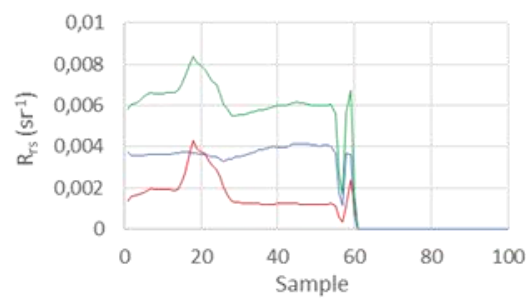

(d)

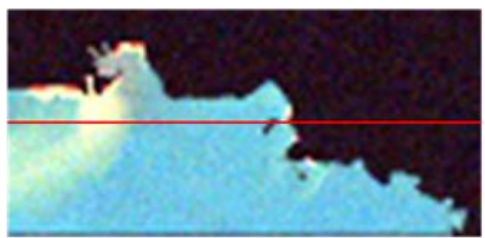

(b)

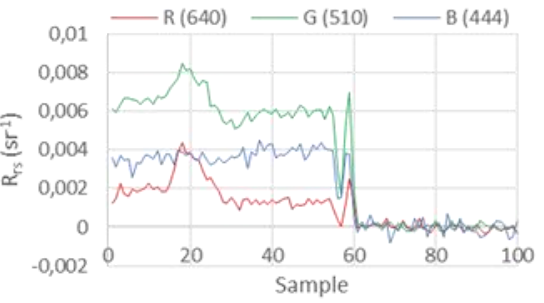

(e)

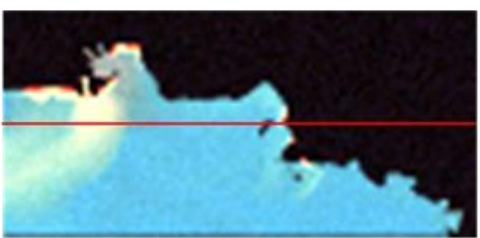

(c)

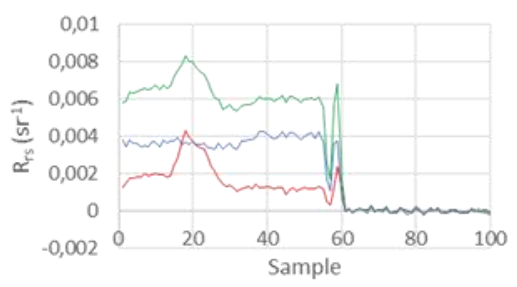

(f)

Figure 5: FCI simulated images at 2:00 PM without noise simulation (a), with noise simulation (b), after noise filtering (c) and the 3 corresponding profiles (d), (e), and (f).

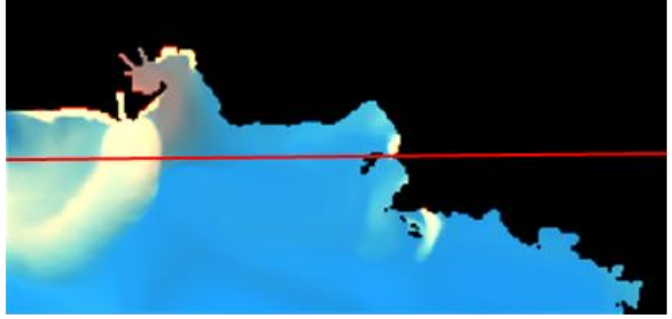

(a)

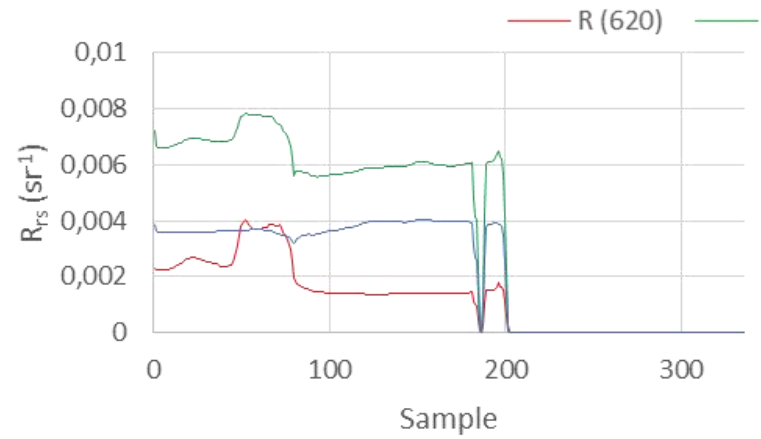

(c)

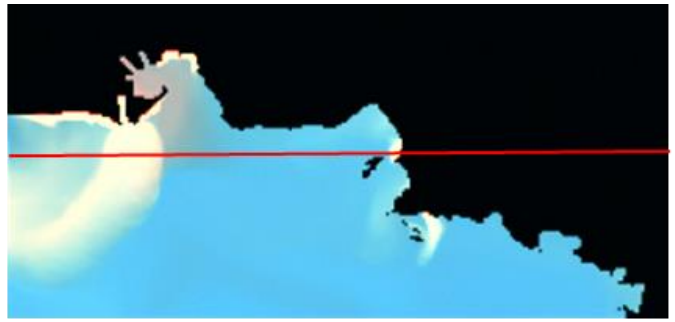

(b)

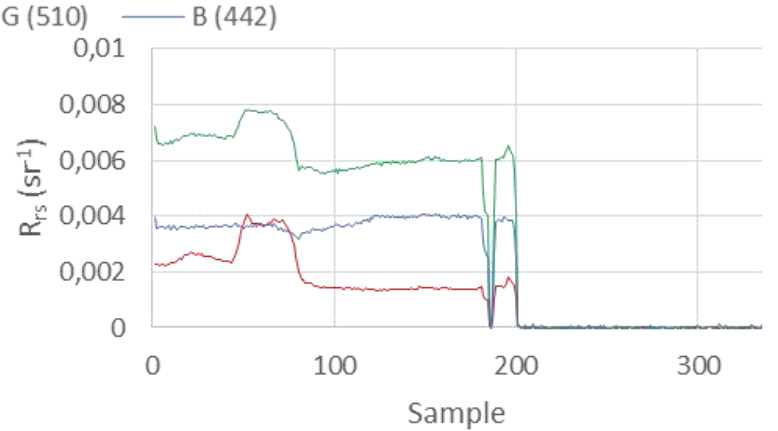

(d)

Figure 6: OLCI simulated images at 10:00 AM without noise simulation (a) and with noise simulation (b) and the 2 corresponding profiles (c) and (d). 


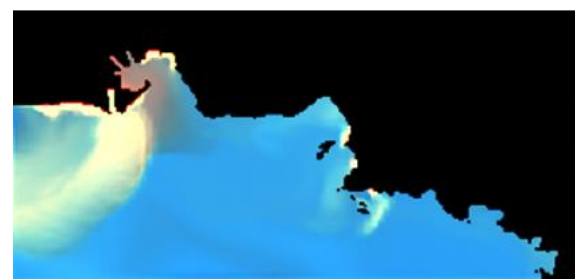

(a)

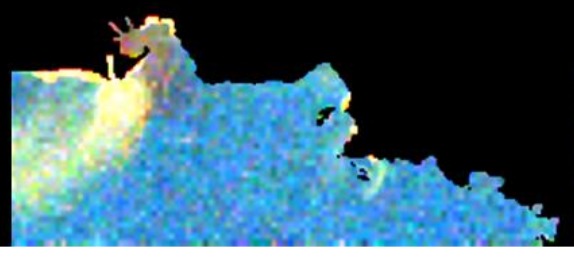

(b)

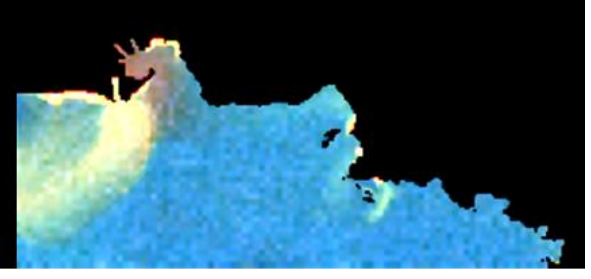

(c)

Figure 7: Fusion product of FCI and OLCI images at 1:00 PM without noise simulation (a), with noise simulation (b), with noise filtering (c).

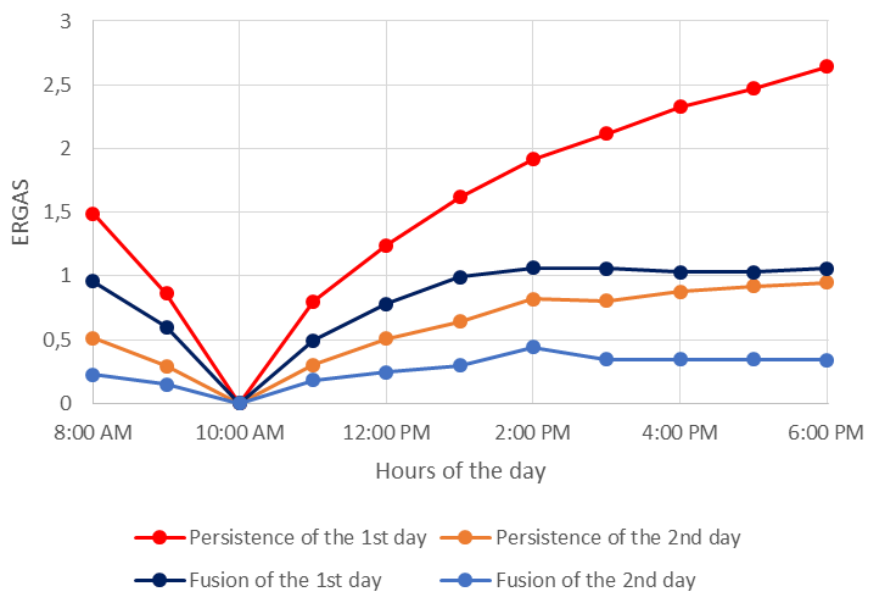

Figure 8: ERGAS index for the SSTF method for the 18/05/2008 and the 19/05/2008 without noise simulation and the corresponding persistence. 


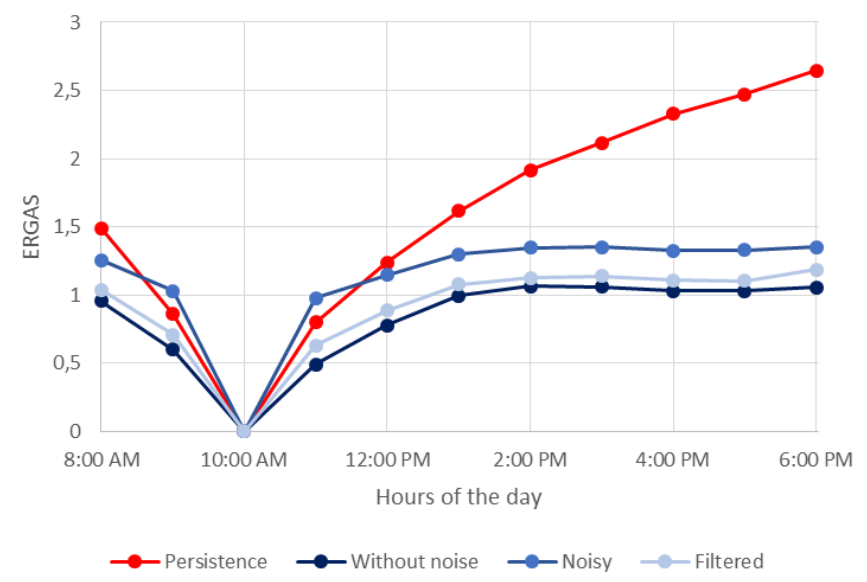

Figure 9: ERGAS index for the SSTF method for the 18/05/2008 without noise simulation, with noise simulation and with noise filtering and the persistence.

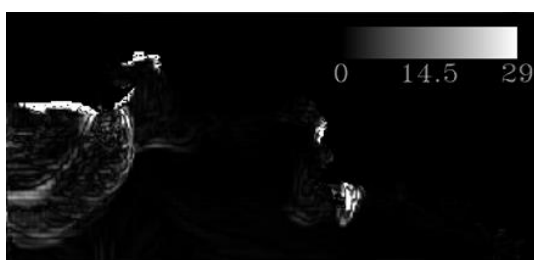

(a)

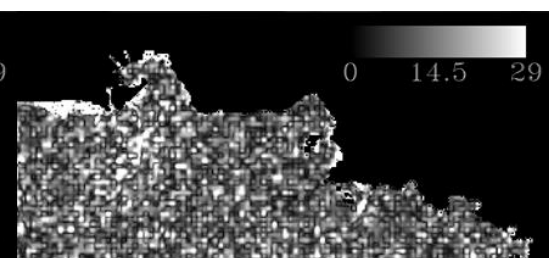

(b)

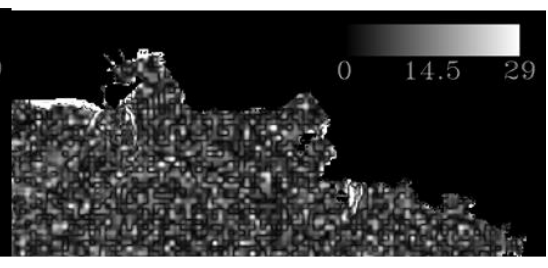

(c)

Figure 10: Averaged error maps on the fusion product at 5:00 PM without noise simulation (a), with noise simulation (b) and with noise filtering (c).

$\mathrm{Chl}$

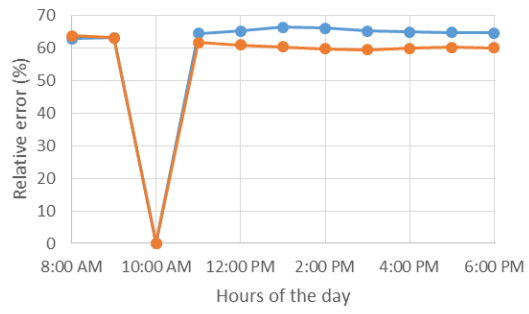

(a)
SPM

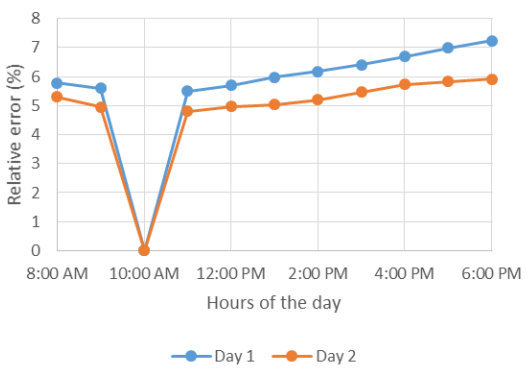

(b)

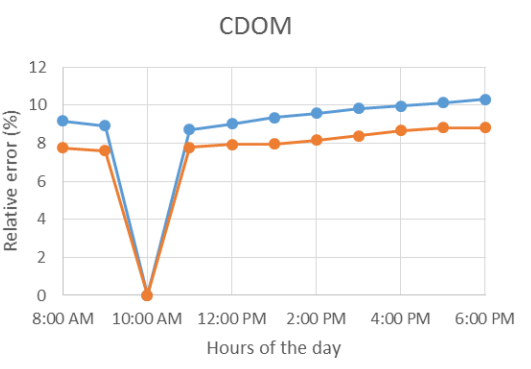

(c)

Figure 11: Relative error between input maps and estimated maps from the fusion products for the 18 and 19/05/2008. 

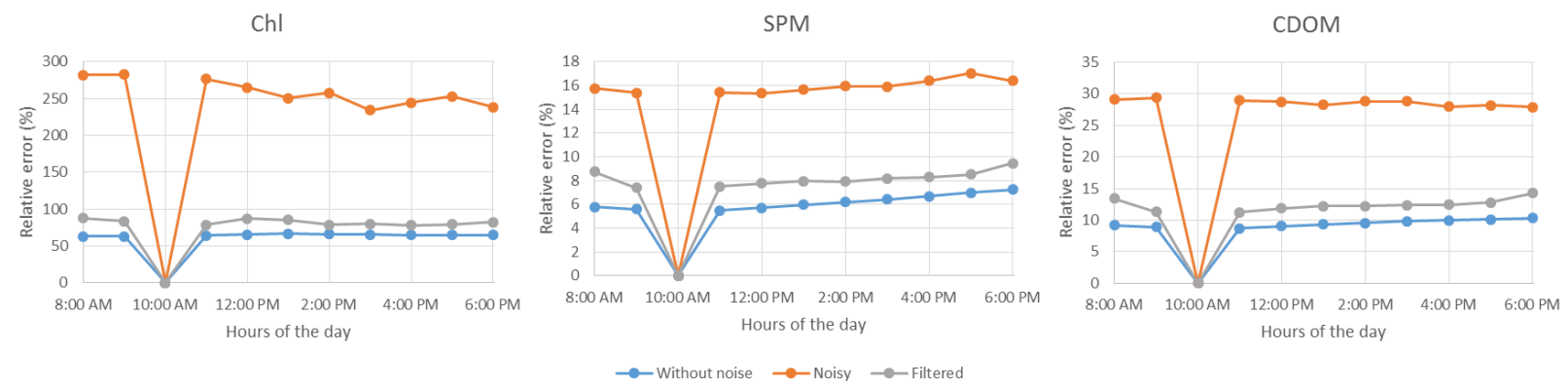

Figure 12: Relative error between input maps and estimated maps from the fusion products for the 18/05/2008 with FCI and OLCI images without noise simulation, with noise simulation and with noise filtering as inputs. 